\title{
Deployment of electric vehicle charging infrastructure. Application to the city of Madrid.
}

\author{
Alexandre DARCHERIF ${ }^{l}$, Bilal AMGHAR ${ }^{2}$, Abdelkrim DOUFENE ${ }^{3}$, and Alain JAAFARI ${ }^{2}$, \\ ${ }^{1}$ Bonzer Inc, MA 02142 Cambridge , USA \\ ${ }^{2}$ ECAM-EPMI, Quartz-Labs, 95000 Cergy France \\ ${ }^{3}$ MIT, MA 02142 Cambridge, USA
}

\begin{abstract}
Most of the scientific and economic observers expect electric vehicles (EVs) to reach more than one-third of new vehicle sales, by 2022 [1]. This growth will be even more pronounced in 2040, when it's expected that more than 40 million of electric vehicles will be sold each year worldwide, leading to a considerable need for electrical energy (the equivalent of the production of about twenty nuclear power plants). This growth cannot be ensured if we do not radically transform our power supply technologies (new generations of batteries, new engines, new supply strategies, etc.) especially since the principal materials for batteries are projected to disappear in the next thirty years. Fortunately, new technologies for charging EVs are appearing (fast charging stations, inductive charging stations), which could help to reducing the need for larger batteries. However, these technologies require significant investment and heavy urban redevelopment. It is therefore important to find a way to optimize these investments both economically and technologically. In this paper, we will focus on electric vehicles and propose a model to optimize the urban infrastructure planning of energy supply stations. we have developed a new approach of infrastructure optimization based on battery charging platform existing technologies and their location in a city. A decision support tool applied to territorial planning is developed in this study.
\end{abstract}

Keywords - Battery Charging Station, Electrified Road Section, Optimization, Electric Vehicle, Network flow, Computer Aided Design (CAD) Tool.

\section{Introduction}

The steady increase in oil prices and awareness regarding environmental issues posed by emissions of Carbon Dioxide $(\mathrm{CO} 2)$ are promoting the current interest in Electric Vehicles (EVs). More and more companies are looking to create the adequate ecosystem to support the future flow of EVs by focusing on solving the major EVs problem, their range. To solve these issues, they brought their attention on the EVs battery charging infrastructure. Three main Battery charging platforms exist: the plug-in charging station, the stationary inductive station, and finally the dynamic charging platform commonly named electrified road section. However, these infrastructures require a huge investment both financial and technologic and are diversified. In a recent research paper (chale et al., 2014 IEEE EnergyCon) it has been addressed how the contribution of autonomy calculation and network modeling help EVs' drivers to enhance their utilization of EVs in a good way, by reducing time waste and cost. Indeed, beyond the capacity of the current batteries, the drivers of
EVs could plan, a priori, their different missions or trips through a simple HMI. However, no study designed a model to optimize the infrastructure's location. There are a lot of issues to be addressed. We focus in the present study on the contribution of network problem solving in order to optimize the choices and construction of battery charging platforms by selecting the best locations based on traffic data, cost, and return on investment of these platforms. However, these data aren't the only constraints. These infrastructures are designed to last; therefore, we need to ensure that our model optimize the infrastructure for the current cities' needs but also anticipate their future needs. In fact, In the city of tomorrow, space will be a fundamental aspect and every centimeter will have to be used at its full potential. The technologies that we will incorporate in our model must therefore be carefully evaluated based on their cost, their durability, and the space they take.

\section{Technology background}

Corresponding author: a.darcherif@gmail.com 
A battery electric vehicle (BEV) is a type of electric vehicle (EV) that uses electrochemical cells commonly named battery. BEVs use electric motors instead of internal combustion engines for propulsion. As per today, we are capable of charging a BEV when parked at a station (Stationary charging) or when it is being driven on a specific road (Dynamic charging).

In a stationary charging platform, a battery can be refilled by plugging an electric cable in the BEVs dedicated port (figure1) or by using induction, while the dynamic charging only allows inductive charging. Every electric car manufacturer has created its own version of stationary or dynamic charging platform. For the sake of clarity, we will cover the common ground of all these technologies as the underlying process is the same for all of them.

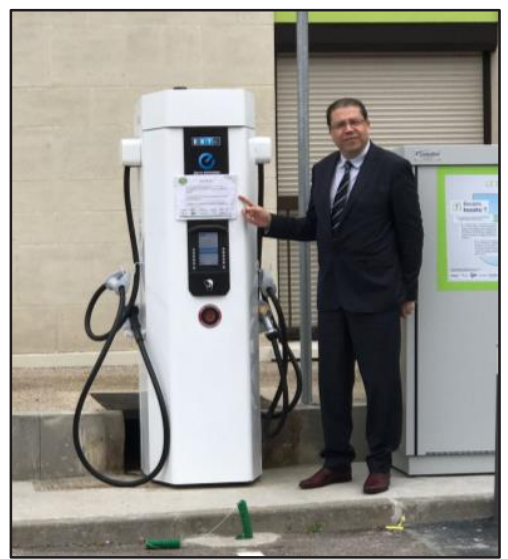

Fig. 1. Fast plug-in charging platform

While the world revolves around plug-in charger, the principle around inductive charging might not be common knowledge. Inductive charging (also known as wireless charging) uses an electromagnetic field to transfer energy between two objects through electromagnetic induction. This is usually performed via a charging pad (figure 2).

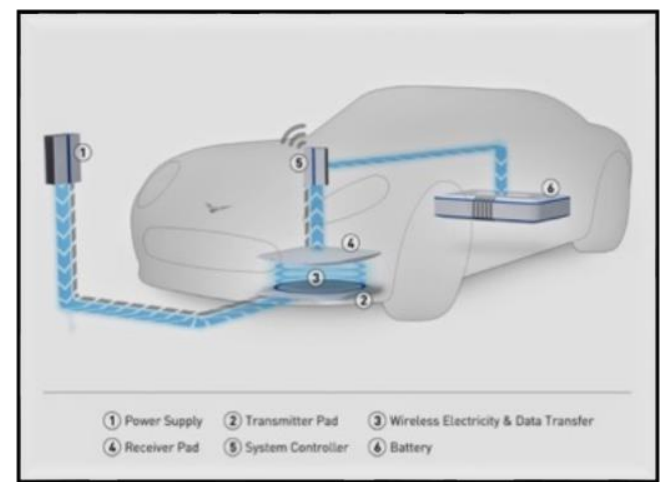

Fig. 2. Inductive battery charging platform

Plug-in and inductive charging platform are two ways of charging a BEVs' battery on a stationary platform. There are pros and cons for both these two systems. In fact, plug-in charging is an easy solution to deploy and relatively well known and supported by every electric vehicle. However, these platforms come with a high maintenance cost because they are subject to vandalism, weather condition, and intensive use. (figure 3). On the other hand, inductive platforms are more expensive, but come with an increased durability, protected connections, weather proof, and more reliable since it can be operated automatically without dependence on people to plug and unplug. The plug-in charging platform also takes a lot of space and might not be compatible with the city of tomorrow where we would have dedicated road for pedestrian and other for car, eliminating the need for pavement and thus making impossible the deployment of plug-in station as they are conceived today.
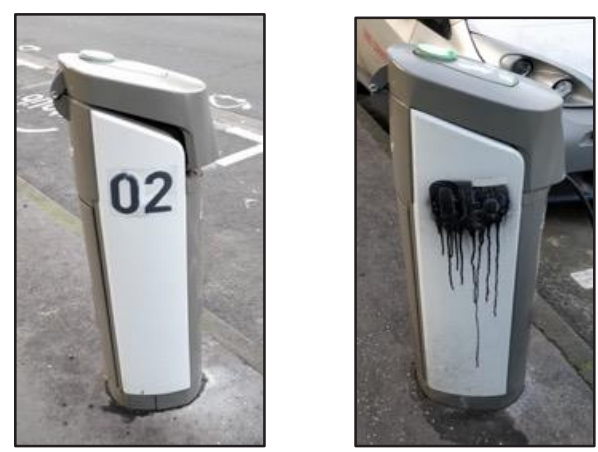

Fig. 3. Two plug-in platforms that has been subject to vandalism in France

Although the inductive charging seems more beneficial and fitted for the concept of the city of tomorrow, we are still in a period of transition and need to take advantage of the most mature technology, the plug-in charging method for the stationary charging. Therefore, the model will incorporate both charging methods. It is to be expected that our model will introduce a way to choose between these two methods.

The dynamic charging technologies revolves around the induction phenomenon. In fact, it has been demonstrated since 2009 by Researchers at the Korea Advanced Institute of Science and Technology (KAIST) that the induction phenomenon is suitable for dynamic charging. They have developed an electric transport system (called Online Electric Vehicle, OLEV) where the vehicles draw power from cables underneath the surface of the road via noncontact magnetic charging (where a power source is placed underneath the road surface and power is wirelessly picked up on the vehicle itself). The dynamic charge will be based on the inductive charging method.

In the following sections, we will detail how we built a battery charging station/road section location optimization model for the city of Madrid.

\section{Background of the study}

With 3.2 million residents and $1.8 \mathrm{~m}$ cars, Madrid often suffers from bad bouts of pollution. In 2015, Madrid registered the second highest number of stationary charging platform in Spain. Since 2011, Spain incorporated consumer rebates toward the purchase of new electric vehicles (EVs) 
into the state's annual budget for the first time. These rebates, which will offer buyers as much as $25 \%$ of the price for a battery-only electric vehicle, a plug-in hybrid, or a fuelcell EV, will help Spain's goal: the reduction of greenhouse gas emissions (GHG emissions) by the 2050-time horizon by between $80 \%$ and $95 \%$ compared to 1990 . Spain has made a major effort towards decarbonization in recent years and is on track to meet the 2020 targets. Given that transportationrelated emissions account for more than one-third of Madrid's greenhouse gas pollution, these incentives can put the state on the right path toward cleaner air, a more stable climate, and a much-needed transition to cleaner vehicles.

In order to help Madrid to reach its goals, we present in this paper a case study of the city of Madrid using our model as explained in the following section.

\section{Methodology}

Our model employs a graph-theoretic framework. We choose to model our problem with a Multi Integer Linear Programming approach. Linear programming (LP) (also called linear optimization) is a method to achieve the best outcome (such as maximum profit or lowest cost) in a mathematical model whose requirements are represented by linear relationships. Linear programming is a special case of mathematical programming (mathematical optimization).

More formally, linear programming is a technique for the optimization of a linear objective function, subject to linear equality and linear inequality constraints.

Linear programs are problems that can be expressed in canonical form as

$$
\text { Maximize } c^{T} x
$$

Subject to $A x \leq b$

$$
\text { And } x \geq 0
$$

Where $\mathrm{x}$ represents the vector of variables (to be determined), $\mathrm{c}$ and $\mathrm{b}$ are vectors of coefficients, $\mathrm{A}$ is a (known) matrix of coefficients, and (.) $\mathrm{T}$ is the matrix transpose. The expression to be maximized or minimized is called the objective function (cTx in this case). The inequalities $\mathrm{Ax} \leq \mathrm{b}$ and $\mathrm{x} \geq 0$ are the constraints which specify a convex polytope over which the objective function is to be optimized.

This approach is ideal when the problem require to take binary decision which is the case in our case study. In our system, the binary decision would be the nature of a node or arc. Either it is a station, either it is not. Obviously, the model will also determine which type of station it is (plugin, inductive, or electrified road for arcs).

\section{Case study}

We defined in the previous sections that the system should take into account constraints such as the creation cost of the platform, their durability, and the space they will occupy. A way to homogenize these constraints is to consider the cost associated with each of these attributes. Hence the durability of a platform will be associated with its maintenance cost, the space will be quantify using the price per square meter of a given location, and the creation of a platform will be quantified by the initial cost one has to pay to build the platform. The goal will therefore be to optimize the resulting equation. Thus, the problem is a problem of maximization of ROI. One of the many advantages to consider this problem from a monetary perspective is that we will be able to compare different pricing method. In fact, some cities might want to generate revenue based on the utilization of the platform or via a recurrent payment. This will inevitably change the landscape of the EVs platform, and it is important for cities to be able to compare both (or more) scenario.

Our study is divided in 4 steps, the collection of data, the mapping of the city, the definition of the constraints, and finally the study of the result.

The data we used are public available information displayed online by the city of Madrid. Obviously, the data collection is an important step of every project and will determine the quality of the result, therefor there is a real need for collaboration with the cities.

As stated earlier, the second step is the mapping of our city in a network format. For this case study, we will use the city of Madrid. The figure below illustrates the center of Madrid.

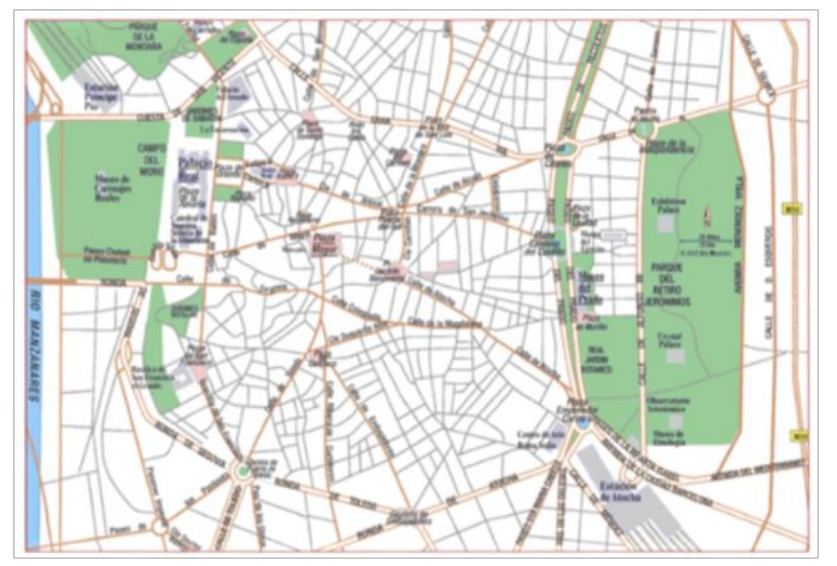

Fig. 4. Road Map of the city of Madrid

The figure below illustrates our resulting network model after the arbitrary mapping of Madrid. 


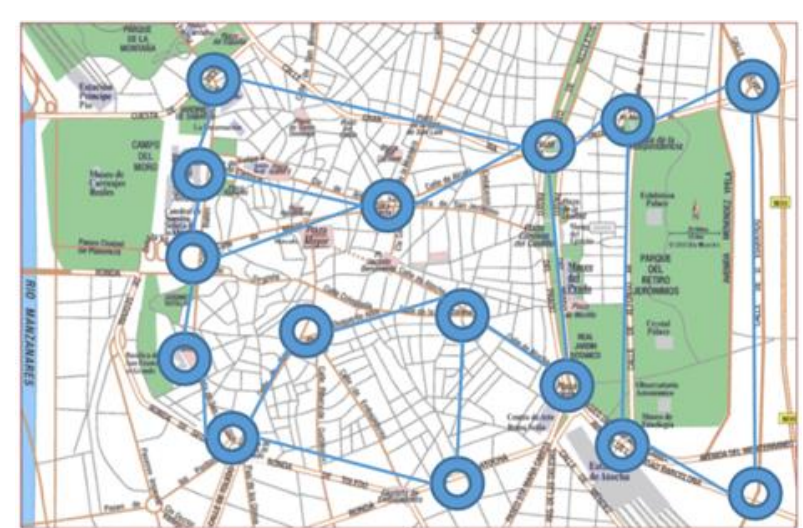

Fig. 5. Representation of the Madrid Road Map

Each node represents a potential spot for a stationary battery charging platform while every arc represents a potential dynamic battery platform.

A network is to some extend a simple square actor-byactor matrix. If the matrix as a whole is called $\mathrm{A}$, then the contents of any given cell are denoted Aij. For instance, in our network, Abuffalo-syracuse would be equal to 1 , because Buffalo is linked to Syracuse. Note that this matrix is not quite symmetric (Aij not always equal to Aji).

It is important to note that the more precise the mapping will be the closer to the reality our system will be. However, this come with a cost for the simple fact that our model needs data for every node and arcs.

According to our methodology we set up the following problem:

$$
\begin{aligned}
\text { Max } f: & \sum_{i, j}^{n}\left(P_{i j} T-C_{i j}-M_{i j} T\right) x_{i j} \\
& +\sum_{i}^{n}\left(P_{i} T-D_{i}-N_{i} T\right) y_{i} \\
& +\sum_{i, j}^{n}\left(R_{i} T-E_{i}-O_{i} T\right) z_{i}
\end{aligned}
$$

Where:

- $\quad \mathrm{P}_{\mathrm{ij}}$ is the profit on the $\operatorname{arc}(\mathrm{i}, \mathrm{j})$;

- $\mathrm{C}_{\mathrm{ij}}$ is the cost of creation of a platform on the arc $(\mathrm{i}, \mathrm{j})$;

- $\quad \mathrm{M}_{\mathrm{ij}}$ is the maintenance cost of a platform on the arc $(\mathrm{i}, \mathrm{j})$;

- $\quad \mathrm{T}$ is the duration of the project. Meaning how long a study wants the project to last or can wait to see result. It is a very important parameter if they receive a certain amount of subvention over a given period of time;

- $\quad \mathrm{x}_{\mathrm{ij}}$ a binary: 1 if we construct a platform on the arc $(\mathrm{I}, \mathrm{j}), 0$ if we don't;

- $\quad y_{i}$ a binary: 1 if we construct a plug-in stationary platform on the node I, 0 if we don't.
- $\quad \mathrm{Z}_{\mathrm{i}}$ a binary: 1 if we construct an inductive stationary platform on the node I, 0 if we don't.

- $\mathrm{Q}_{\mathrm{i}}$ and $\mathrm{R}_{\mathrm{i}}$ are the profit on the node I;

- $\mathrm{D}_{\mathrm{i}}$ and $\mathrm{E}_{\mathrm{i}}$ are the cost of creation of a platform on the node I;

- $\mathrm{N}_{\mathrm{i}}$ and $\mathrm{O}_{\mathrm{i}}$ represent the maintenance cost of a platform on the node I;

Subject to the following constraints:

- $\quad \sum \mathrm{x}_{\mathrm{ij}} \geq 0$

- $\quad \sum y_{i}+z_{i} \geq 0$

- If $y_{i}=1$ then $z_{i}=0$

- If $\mathrm{z}_{\mathrm{i}}=1$ then $\mathrm{y}_{\mathrm{i}}=0$

- $\quad \sum \mathrm{C}_{\mathrm{ij}} \mathrm{x}_{\mathrm{ij}}+\sum \mathrm{D}_{\mathrm{i}} \mathrm{y}_{\mathrm{i}} \leq$ Investment

- $\quad\left(\sum \mathrm{P}_{\mathrm{ij}} \mathrm{x}_{\mathrm{ij}}+\sum \mathrm{Q}_{\mathrm{i}} \mathrm{y}_{\mathrm{i}}\right)-\left(\sum \mathrm{M}_{\mathrm{ij}} \mathrm{x}_{\mathrm{ij}}+\sum \mathrm{N}_{\mathrm{i}} \mathrm{y}_{\mathrm{i}}\right) \geq$ Minimum acceptable profit or loss

In the definition of the problem, we assumed that we will consider situations where our infrastructure has a positive ROI and the cost of infrastructure is covered by the initial investment. There are other versions of our model where we accept loss, and/or we assume that the initial investment plus the profit should cover the creation cost.

To solve the problem, we created a model using GLPK. The GLPK (GNU Linear Programming Kit) package is intended for solving large-scale linear programming (LP), mixed integer programming (MIP), and other related problems. It is a set of routines written in ANSI $\mathrm{C}$ and organized in the form of a callable library. Our model allows us to compute multiple instance of the same problem with different constraints. This model is integrated in a web application that allow us to upload data and constraints and keep the result in a database for comparisons. The web tool is built in such a way to be as user-friendly as possible as the main users will be cities council or investor that look forward building battery charging platforms.

The following part display the impact of the investment on the problem. The problem being a ROI maximization problem, the Investment is a critical factor and has an important weight on the output.

In the following figures, we took the previously designed mapping of Madrid. Each node represents a potential spot for a stationary battery charging platform while every arc represents a potential dynamic battery platform.

- A dynamic platform is represented by a green link between two nodes;

- A stationary charging platform is represented by a green circle on a node regardless if it is a plug-in or an inductive platform;

- The non-chosen nodes or arcs are in blue. 


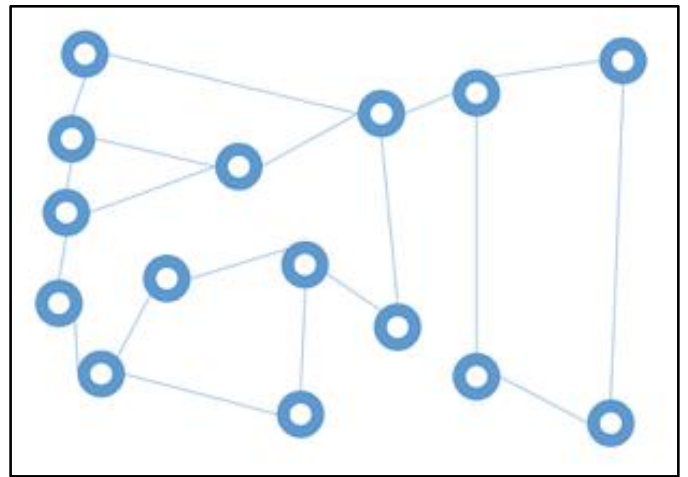

Fig. 6. Result with Investment $=\$ 0$

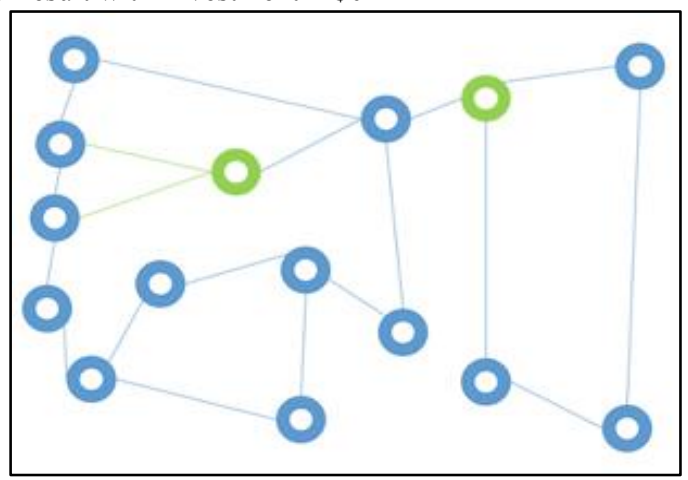

Fig. 7. Result with Investment $=\$ 500 \mathrm{M}$

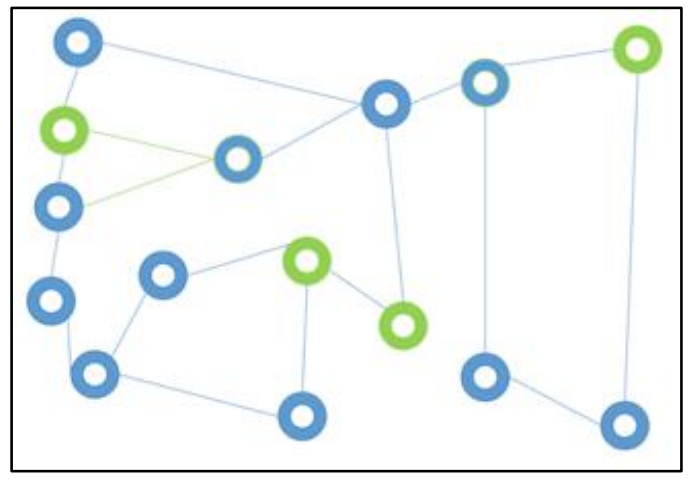

Fig. 8. Result with Investment $=\$ 1 B$

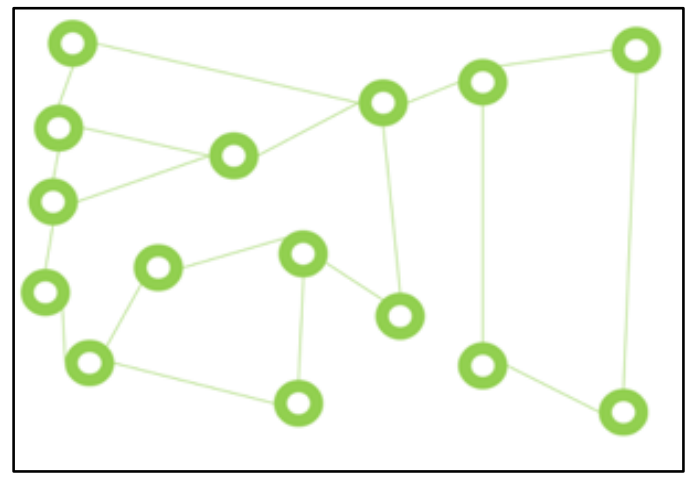

Fig. 9. Result with Investment $=\$ 100 \mathrm{~B}$

The investment is the defining constraint. One interesting conclusion is that the output is not linear. If we observe the figure 4 and 5 , we can conclude that an increase of the investment does not necessarily mean an increase of infrastructure, but rather a reallocation of the resource therefor, when we need to be careful when defining the investment.

The figure below is the result we get for an Investment of $\$ 250 \mathrm{M}$ in the city of Madrid. Obviously, the results are based on the data collected and the constraints used, therefor it is optimized for our conception of the city of Madrid. If we had access to all the data that our system needs, we would find completely different result.

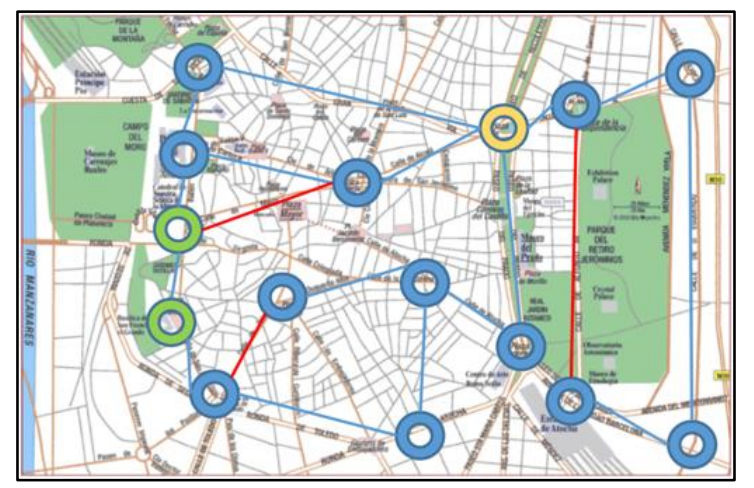

Fig. 10. In green the plug-in battery charging platform; In yellow the inductive stationary platforms; In red, the electrified roads (Dynamic platforms)

\section{Perspectives}

The relevance of our model has been demonstrated for cities' initiative. However, this model could also be used by companies that want to leverage the creation of battery charging platform for their business. This is the case of Bonzer Inc, an electric car sharing company where the battery charging platform also happen to be parking spot for their cars. Thanks to its proximity with MIT, Bonzer Inc is committed to help the building of smart cities, therefore it is mandatory that their expansion strategy fit with the concept of smart city. Unlike most of the car sharing companies, Bonzer Inc enables the user to book and access the car from a smartphone. While this is certainly more user-friendly than using a membership card, the real advantage for our study lies in the fact that we can feed our model with more data. The starting point, the destination, the cost of the trip, and the profile of the user, after being anonymized, could all be used to optimize the location of battery charging platform for Bonzer Inc. 


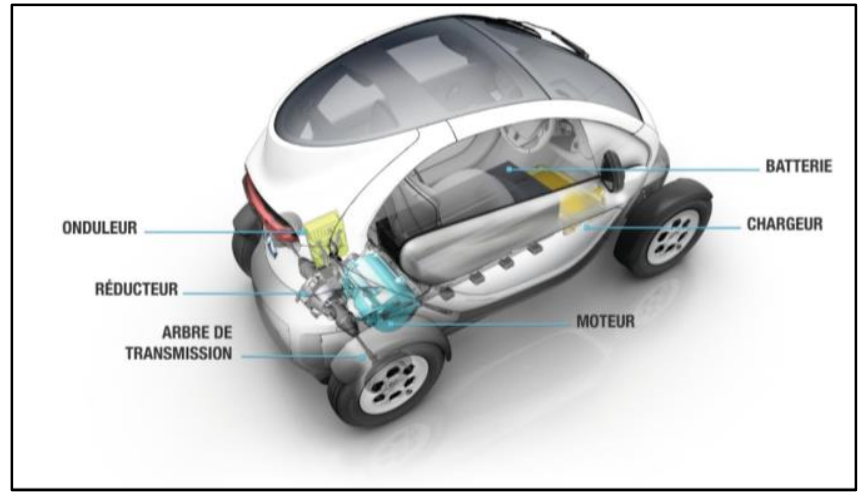

Fig. 11. Bonzer Inc car anatomy

\section{Conclusion}

The model developed in this paper is an original contribution to the design and development of static and dynamic charging infrastructures for electric vehicles of tomorrow. It is a cornerstone on the long road to the sustainable deployment of "the city of the future".

The model applies to both new and extended works. For the sake of convenience, we have considered the case of a new work here.

Our work aims to support the development of new technological solutions for electric mobility (smart roads of tomorrow). the goal is to offer the driver a high level of comfort in the use of his vehicle. Thus, the driver would never need to stop at a filling / charging station. The design and engineering of cars would change drastically as large engines, fuel tanks and batteries would no longer be needed. Reducing our dependence on fossil fuels would of course be a savior for the environment. The electric road would be a network in the computer sense of the word; potentially allowing all kinds of vehicle tracking, autonomous driving, vehicle-to-vehicle networks, intelligent braking, and more.
After studying the habits of the average driver and using our model in multiple cities, our first hypothesis would be to position dynamic battery charging platform on roads subject to high traffic. By electrifying these roads, we ensure the fact that EV will have enough battery to drive in the city and outside the city. This means that we will require less stationary charging platforms in big cities.

\section{References}

1. European Energy and Transport - Trends to 2030. European Commission- Directorate-General for Energy and Transport. 2007.

2. Final Energy Consumption by Sector 2010. Official document of European Environment Agency, available online_http://www.eea.europa.eu/dataand-maps/indicators/transport-finalenergyconsumption-by-mode/2010 .

3. Takuto Ishimatsu, Abdelkrim Doufene. Abdullah Alawad and Olivier L. de Weck. Desalination Network Model Driven Decision Support System: A Case Study of Saudi Arabia. in The International Desalination Association World Congress on Desalination and Water Reuse. San Diego, CA, USA, August 2015. 